\title{
A 52-year-old Female With Ramsay Hunt Syndrome
}

\author{
Ketki Soin, MSIII, Eric Struble, MD and Whitney Jackson, MD
}

\section{Case Report}

A 52 year-old female with a history of poorly controlled type II diabetes and a recent admission of right-sided pre-septal orbital cellulitis presented with a facial rash and severe ear and eye pain for three days. The patient noted sudden right-sided vision loss and associated right-sided face, ear and eye pain three days prior to admission. The pain was a $9 / 10$ and was worsening since its onset. Upon questioning she also noted decreased taste sensation. She denied tinnitus, vertigo, hearing loss, nausea, vomiting, or fevers.

On physical exam, the patient was afebrile, she had a heart rate of 102 beats per minute, a blood pressure of $122 / 57 \mathrm{mmHg}$, and an oxygen saturation of $94 \%$ on room air. Her skin exam was remarkable for a macular rash on the right side of her face in a dermatomal distribution, extending from her mouth to the external ear and continuing to her neck. She had vesicular lesions on her nose, known as Hutchinson's sign. Her ear exam showed erythema of the right ear, swelling of the pinna and auricle, and multiple vesicles present on her right tympanic membrane and external auditory canal. Her eye exam was significant for no light perception in the right eye with erythema and edema of her right eyelid. Neurological exam was remarkable for cranial nerve II, VI, and peripheral VII palsies. Weber test lateralized to the left ear and the Rhine test was positive bilaterally. On admission, laboratory studies revealed a white blood cell count (WBC) of $10.0 \mathrm{~B} / \mathrm{L}$. The differential included $73.4 \%$ neutrophils, $13.4 \%$ lymphocytes, and $7.6 \%$ monocytes. Other significant studies were a hemoglobin A1c of $13.7 \%$, glucose of $388 \mathrm{mg} / \mathrm{dl}$ and a creatinine level of $1.6 \mathrm{mg} / \mathrm{dl}$.

The patient was admitted and treated with IV acyclovir, IV methylprednisolone, fluids and insulin drip at 4 units/hr was initiated. During her hospital stay, an MRI of the brain and orbits was performed to rule out meningeal involvement. The MRI showed abnormal enhancement of the right optic nerve sheath without meningeal involvement (Figure).

The patient's hospital course was complicated by difficult-tocontrol diabetes. Her glucose levels ultimately normalized with very high insulin requirements. Over the course of her hospitalization, insulin and methylprednisolone were tapered. She was discharged one week after admission once her ear and eye pain resolved and her glucose levels normalized. Despite therapy with acyclovir and corticosteroids, the patient's peripheral facial palsy and vision changes did not resolve in the hospital. She was to follow-up with the neuro-opthalmology office as an outpatient.

\section{Discussion}

The varicella zoster virus is known for two classic clinical syndromes: varicella or chicken pox is the primary infection, while zoster or shingles is the reactivation of latent infection
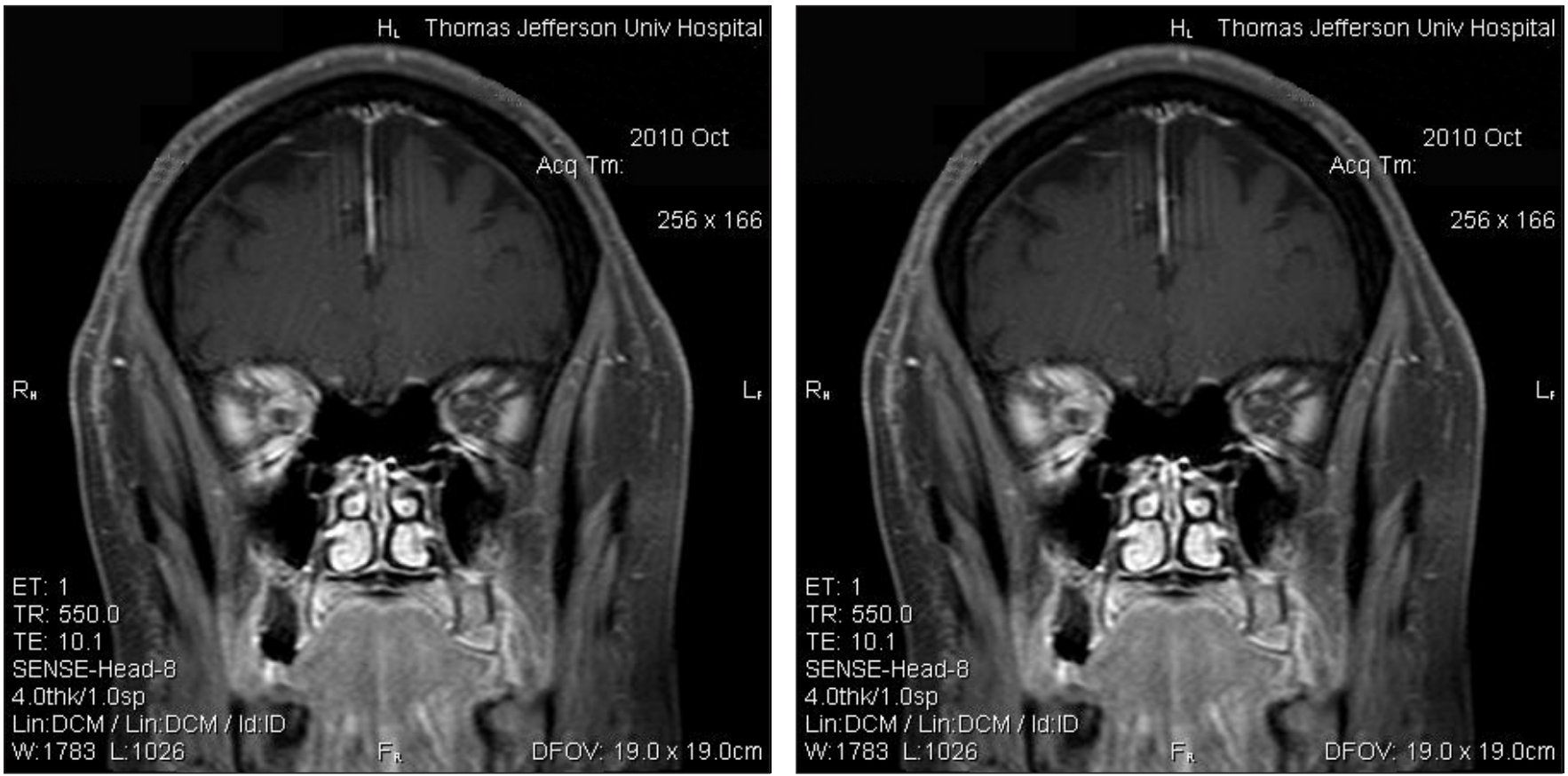

Figure: MRI, brain. Abnormal enhancement of the right optic nerve sheath with surrounding inflammatory enhancement in the adjacent intraconal fat, compatible with the patient's clinical history of herpes zoster and decreased light perception in the right eye. 
in the dorsal root ganglia. However, other complications of herpes zoster exist; these include cranial nerve palsies, herpes zoster ophthalmicus, acute retinal necrosis, aseptic meningitis, encephalitis, or stroke syndromes secondary to cerebral artery infection. Ramsay-Hunt syndrome, herpes zoster oticus, is facial and auditory involvement due to reactivation of herpes zoster in the geniculate ganglion. This rare manifestation of herpes zoster causes painful vesicles in the external auditory canal, loss of taste in the anterior one-third of the tongue, ipsilateral facial palsy, and it may be associated with herpes zoster ophthalmicus, threatening visual loss.

The incidence of Ramsay Hunt syndrome is less than $1 \%$ of all patients with herpes zoster. ${ }^{1}$ In a review of 859 patients with herpes zoster, complications including Ramsay-Hunt, postherpetic neuralgia, superinfection, ocular involvement, Bell's palsy and meningitis were associated with advanced age and comorbidities including diabetes, HIV, cancer and transplant recipients. HIV and transplant recipients present with more complications. ${ }^{2}$

Antiviral agents and corticosteroids are first line therapies for herpes zoster with cranial nerve involvement. Antiviral therapy may improve outcomes by prohibiting progression of the varicella virus and reducing long-term nerve damage. It has even been suggested that antiviral therapy can improve outcomes of facial weakness in Ramsay Hunt syndrome, ${ }^{3}$ however there is little data to support this. In a retrospective review of 26 patients with herpes zoster oticus, all were treated with Acyclovir, with $84 \%$ showing recovery. There was no control group; age and diabetes mellitus were identified as poor prognostic factors. ${ }^{4}$

Steroids, presumably, are also beneficial in treatment in that they have anti-inflammatory effects, which limit neural edema and subsequently, tissue damage and cell death. It is therefore logical that both steroids and antiviral treatment together should be used to treat zoster. In a study by Kinishi and colleagues, 91 patients were treated with acyclovir and corticosteroids compared to 47 patients treated with corticosteroids alone. Voluntary facial movement recovered in 82 of the 91 patients $(90 \%)$ treated with both acyclovir and corticosteroids compared to 30 of the 47 patients (64\%) treated with corticosteroids alone. ${ }^{5}$
Timing of treatment also seems to play a critical role in recovery Ramsey Hunt patients. In an observational study of 81 patients, administration of both steroids and acyclovir within 3 days of onset of symptoms showed full recovery of deficits in 21 out of 28 patients $(75 \%)$. When treatment was initiated between days 4 and 7 of symptom onset, 14 of 29 patients (48.3\%) showed absolute resolution. For those that received treatment after 7 days, full recovery was achieved in only 7 of 23 patients $(30.4 \%){ }^{6}$

However, this data has not been convincingly replicated. Recent Cochrane reviews question the role of acyclovir, other antivirals, and corticosteroids in herpes zoster oticus treatment and outcomes. Only one randomized controlled trial was included in the Cochrane Review looking at antiviral therapy vs antiviral therapy with corticosteroids for treatment of Ramsay-Hunt syndrome. It included only 15 patients and there was no statistically significant difference between the two groups. ${ }^{3}$ No randomized, controlled trials exist looking at corticosteroid treatment for Ramsay Hunt. ${ }^{7}$ After thorough literature review, they conclude that there is currently insufficient randomized, controlled trial data to provide evidence for or against standard antiviral therapy alone or in combination with steroids for the treatment of Ramsay Hunt syndrome.,

\section{References}

1. Whitley RJ. A 70-year-old woman with shingles: review of herpes zoster. JAMA 2009;302:73-80.

2. Galil K, Choo PW, Donahue JG, Platt R. The sequelae of herpes zoster. Arch Intern Med 1997;157:1209-13.

3. Uscategui T, Doree C, Chamberlain I, Burton M. Antiviral therapy for Ramsay Hunt syndrome (herpes zoster oticus with facial palsy) in adults. Cochrane Database of Systematic Reviews 2008, Issue 4.

4. Yeo SW, Lee DH, Jun BC, Chang KH, Park YS. Analysis of prognostic factors in Bell's palsy and Ramsay Hunt syndrome. Auris Nasus Larynx 2007;34:159-64.

5. Kinishi M, Amatsu M, Mohri M, Saito M, Hasegawa T, Hasegawa S. Acyclovir improves recovery rate of facial nerve palsy in Ramsay Hunt syndrome. Auris Nasus Larynx 2001;28:223-6.

6. Murakami S, Hato N, Horiuchi J, Honda N, Gyo K, Yanagihara N. Treatment of Ramsay Hunt syndrome with acyclovir-prednisone: significance of early diagnosis and treatment. Ann Neurol 1997;41:353-7.

7. Uscategui T, Doree C, Chamberlain I, Burton M. Corticosteroids as adjuvant to antiviral treatment in Ramsay Hunt Syndrome (herpes zoster oticus with facial palsy) in adults. Cochrane Database of Systematic Reviews 2009, Issue 1. 